\title{
DNA methylation and leukemia susceptibility in China: Evidence from an updated meta-analysis
}

\author{
DANJIE JIANG ${ }^{1 *}$, YIRUN LI $^{1 *}$, QINGXIAO HONG ${ }^{1}$, YUSHENG SHEN $^{1}$, CHUNJING XU $^{1}$, \\ YAN XU ${ }^{1}$, HUANGKAI ZHU ${ }^{1}$, DONGJUN DAI ${ }^{1}$, GUIFANG OUYANG ${ }^{2}$ and SHIWEI DUAN ${ }^{1}$ \\ ${ }^{1}$ Medical Genetics Center, School of Medicine, Ningbo University, Ningbo, Zhejiang 315211; \\ ${ }^{2}$ Department of Hematology, Ningbo First Hospital, Ningbo, Zhejiang 315010, P.R. China
}

Received November 14, 2014; Accepted May 20, 2016

DOI: $10.3892 / \mathrm{mco} .2016 .959$

\begin{abstract}
Mounting evidence supports a role for DNA methylation in the pathogenesis of leukemia; however, there no overview of these results in the Chinese population. The present study performed a comprehensive meta-analysis to establish candidate genes with an altered methylation status in Chinese leukemia patients. Eligible studies were identified through searching the National Center of Biotechnology Information PubMed and Wanfang databases. Studies were pooled and overall odds ratios with corresponding confidence intervals were calculated. A total of 4,325 leukemia patients and 2,010 controls from 94 studies on 53 genes were included in this meta-analysis, and 47 genes were found to be aberrantly methylated in leukemia patients. A further subgroup meta-analysis by leukemia subtype demonstrated that hypermethylation of 5 genes, namely cyclin-dependent kinase $(C D K N) 2 A$, DNA-binding protein inhibitor-4, $C D K N 2 B$, glioma pathogenesis-related protein 1 and $p 73$, contributed to the risk of various subtypes of leukemia. In addition, a strong association between $C D K N 2 A$ and leukemia was identified in Chinese $(\mathrm{P}<0.00001)$ but not in European patients. The aberrantly methylated genes identified in the present meta-analysis may help elucidate the mechanisms underlying the development of leukemia in Chinese patients.
\end{abstract}

Correspondence to: Dr Shiwei Duan, Medical Genetics Center, School of Medicine, Ningbo University, 818 Fenghua Road, Ningbo, Zhejiang 315211, P.R. China

Email: duanshiwei@nbu.edu.cn

Dr Guifang Ouyang, Department of Hematology, Ningbo First Hospital, 59 Liuting Road, Ningbo, Zhejiang 315010, P.R. China Email: ouyangguifang2000@aliyun.com

*Contributed equally

Key words: leukemia, DNA methylation, epigenetics, ethnic difference, Chinese population

\section{Introduction}

Leukemia is a complex hematological malignancy, characterised by clonal proliferation of malignant hematopoietic stem cells in the blood and bone marrow (1), with a total of 350,000 new cases and 25,700 deaths annually (2). Genetic as well as environmental factors have been suggested to be associated with leukemia, including trisomy 21, gender, cytotoxicity of anticancer drugs, exposure to benzene and ionising radiation (3-6). Leukemia is a heterogeneous disease that comprises acute lymphocytic leukemia (ALL), chronic lymphocytic leukemia (CLL), acute myelogenous leukemia (AML) and chronic myelogenous leukemia (CML). ALL accounts for $81 \%$ of childhood leukemia cases, while CLL and AML frequently occur in adults (7).

Racial and ethnic disparities have been identified in the expression of leukemia-related genes, the clinical outcome and the mortality rate of leukemia (8-12). These disparities are likely due to a combination of genetic, environmental and socioeconomic factors (13), which may affect epigenetic changes. Epigenetics, such as DNA methylation, have been shown to play an important role in cancer susceptibility $(14,15)$. Therefore, DNA methylation studies may help elucidate these racial and ethnic disparities in leukemia patients.

Aberrant DNA methylation of genes has been shown to be associated with a large number of human malignancies $(16,17)$. Although a recent meta-analysis by our group identified significant associations between a number of aberrantly methylated genes and leukemia (18), the majority of the studies published in the Chinese language are overlooked. Thus, the aim of the present study was to focus on the association of aberrant DNA methylation and leukemia susceptibility in the Chinese population and to investigate ethnic differences in DNA methylation using subgroup meta-analyses.

\section{Materials and methods}

Selection of studies. A systematic literature search was performed through the National Center for Biotechnology Information (NCBI) PubMed and Wanfang literature databases, updated until July 10, 2014. The search was performed using the keywords 'leukemia' and 'methylation'. Potentially relevant articles were identified by their titles and abstracts, followed by selection of eligible studies based on full-text analysis. 
Case-control studies on gene methylation in Chinese leukemia patients containing sufficient information on methylation to calculate the odds ratios (ORs) and 95\% confidence intervals (CIs) were considered to be eligible. A flow chart of the study selection process is shown in Fig. 1.

Data extraction. The following characteristics were extracted from each eligible study: First author's name, year of publication, disease category and methylation status of cases and controls. All the studies included were reviewed by three authors (D.J., Y.S. and C.X.). For genes with methylation data in other populations, the corresponding data were retrieved and subjected to meta-analyses for comparison with the Chinese population.

Statistical analysis. Review Manager 5.0 software (The Nordic Cochrane Centre, The Cochrane Collaboration, Copenhagen, Denmark) was used for the meta-analysis. The ORs and 95\% CIs were calculated to evaluate the association between gene methylation and leukemia. Heterogeneity of the included studies was assessed using $\mathrm{I}^{2}$ statistics (19). When there was significant heterogeneity $\left(\mathrm{I}^{2}>50 \%\right)$, the random-effects model was used to calculate the overall OR and 95\% CI; otherwise, the fixed-effects model was applied (20).

\section{Results}

Eligible studies. As shown in Fig. 1, 1,477 potentially relevant studies were identified for initial review from the NCBI PubMed and Wanfang literature databases. A total of 1,380 studies were excluded (1,036 irrelevant studies, 227 non-case-control studies and 117 studies with insufficient data). Finally, a total of 94 studies (61 studies on ALL, 76 on AML, 11 on CLL, 31 on CML and 3 other studies on leukemia), were included in the present meta-analysis (31,38,44-135). A total of 53 genes were identified among 4,325 leukemia patients and 2,010 control subjects, of which 19 were reported by only 1 study, 15 by 2 studies and 19 by $\geq 3$ studies (Tables I and II). For 47 of these 53 genes, aberrant methylation was proved to be significantly associated with leukemia.

Meta-analysis of the association between cyclin-dependent kinase $(C D K N) 2 A$ methylation and leukemia. As shown in Fig. 2, 566 cases and 361 controls were included in the meta-analysis of $C D K N 2 A$ methylation. The results indicated that hypermethylation of $C D K N 2 A$ was a risk factor for leukemia $(\mathrm{P}<0.00001$; OR=19.99; 95\% CI: 11.37-35.17). Subgroup analysis by type of leukemia revealed that hypermethylation of $C D K N 2 A$ was associated with an increased risk of $\mathrm{AML}(\mathrm{P}<0.00001 ; \mathrm{OR}=17.86 ; 95 \% \mathrm{CI}$ : 7.79-40.93 $)$, ALL $(\mathrm{P}<0.00001 ; \mathrm{OR}=24.01 ; 95 \% \mathrm{CI}: 10.23-56.33)$ and CLL ( $\mathrm{P}=0.04$; OR=15.95; 95\% CI: 1.16-218.94), but not of CML $(\mathrm{P}=0.08)$. However, there was no significant difference in the association results among different leukemia types $(\mathrm{P}=0.87)$.

Meta-analysis of the association between CDKN2B methylation and leukemia. The meta-analysis of the association between $C D K N 2 B$ methylation and leukemia included 24 studies comprising 463 cases and 302 controls (Fig. 3).

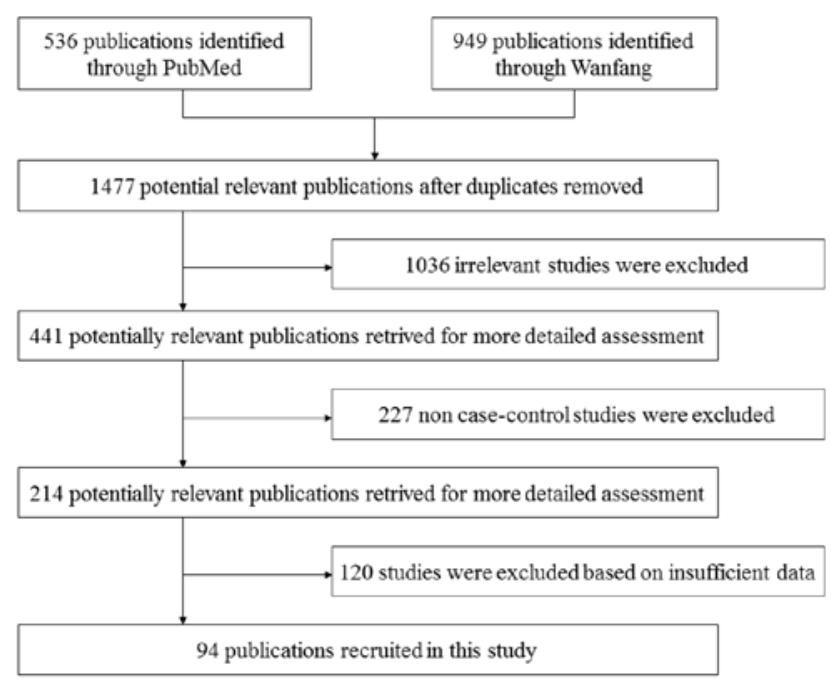

Figure 1. Flow diagram of the study selection process.

The results revealed that hypermethylation of the $C D K N 2 B$ gene was associated with the risk of leukemia $(\mathrm{P}<0.00001$; $\mathrm{OR}=42.45 ; 95 \% \mathrm{CI}=22.98-78.42$ ). These 24 studies included 12 studies on ALL, 10 studies on AML and 2 studies on CML. A subtype meta-analysis revealed that $C D K N 2 B$ promoter methylation was a risk factor for $\mathrm{AML}(\mathrm{P}<0.00001 ; \mathrm{OR}=54.11$; 95\% CI: 21.07-138.93), ALL $(\mathrm{P}<0.00001 ; \mathrm{OR}=35.76$; 95\% CI: 14.92-85.69) and CML $(\mathrm{P}=0.004 ; \mathrm{OR}=27.06$; 95\% CI: 2.88-254.55). There was no significant difference in the association results among different leukemia types $(\mathrm{P}=0.76)$.

Meta-analysis of the association between DNA-binding protein inhibitor-4 (ID4) methylation and leukemia. A total of 10 studies were included in the ID4 methylation analysis (Fig. 4). The meta-analysis revealed that ID4 methylation was a risk factor for leukemia $(\mathrm{P}<0.00001$; $\mathrm{OR}=70.08$; 95\% CI: 24.12-203.64). Hypermethylation of the ID4 gene was associated with an increased risk of AML $(\mathrm{P}<0.00001$; $\mathrm{OR}=116.32 ; 95 \% \mathrm{CI}: 25.40-532.59)$, ALL $(\mathrm{P}<0.00001$; $\mathrm{OR}=104.68 ; 95 \%$ CI: 17.27-634.39), CML ( $\mathrm{P}=0.002 ; \mathrm{OR}=20.17$; 95\% CI: 3.05-133.21) and CLL $(\mathrm{P}=0.002 ; \mathrm{OR}=693.00$; 95\% CI: 11.87-40460.96). There was no significant difference in the association results among different leukemia types $(\mathrm{P}=0.33)$.

Meta-analysis of the association between glioma pathogenesis-related protein 1 (GliPRl) methylation and leukemia. As shown in Fig. 5, the meta-analysis of the association between GliPR I methylation and leukemia included 9 studies. The results revealed that GliPR 1 methylation was a risk factor for leukemia $(\mathrm{P}<0.00001 ; \mathrm{OR}=6.45 ; 95 \%$ CI: 2.88-14.45). Hypermethylation of the GliPRl gene was associated with an increased risk of AML $(\mathrm{P}<0.00001 ; \mathrm{OR}=30.33$; 95\% CI: 15.83-58.11), ALL $(\mathrm{P}<0.0001 ; \mathrm{OR}=3.39$; 95\% CI: 1.88-6.13) and CML ( $\mathrm{P}=0.006$; $\mathrm{OR}=2.49$; 95\% CI: 1.30-4.77). Moreover, there was a significant difference in the association of GliPR 1 hypermethylation with the risk of leukemia among the different subtypes $(\mathrm{P}<0.00001)$. 
Table I. Eligible case-control leukemia studies in the Chinese population.

\begin{tabular}{|c|c|c|}
\hline Gene & No. of studies & Overall OR (95\% CI) \\
\hline$C D K N 2 A$ & 26 & $19.99(11.37-35.17)$ \\
\hline$C D K N 2 B$ & 24 & $42.45(22.98-78.42)$ \\
\hline ID4 & 10 & $70.08(24.12-203.64)$ \\
\hline GliPR1 & 9 & $6.45(2.88-14.45)$ \\
\hline p73 & 7 & $17.07(6.20-47.02)$ \\
\hline$C T$ & 6 & $46.85(12.15-180.65)$ \\
\hline$D A P K$ & 6 & $17.19(5.43-54.41)$ \\
\hline$P R B$ & 6 & $32.10(9.19-112.16)$ \\
\hline SFRP5 & 5 & $11.45(3.19-41.12)$ \\
\hline$I G S F 4$ & 4 & $14.41(3.21-64.72)$ \\
\hline$P R A$ & 4 & $38.11(8.14-178.29)$ \\
\hline RASSF $1 A$ & 4 & $22.62(5.11-100.17)$ \\
\hline$S F R P 2$ & 4 & $30.28(7.18-127.74)$ \\
\hline$L R P 15$ & 3 & $41.45(6.63-259.10)$ \\
\hline RIZ1 & 3 & $9.86(1.84-52.78)$ \\
\hline SFRP4 & 3 & $14.31(2.77-73.90)$ \\
\hline$W T 1$ & 3 & $0.24(0.10-0.54)$ \\
\hline $\mathrm{ZO}-1$ & 3 & 99.65 (18.14-547.54) \\
\hline$R A R A$ & 3 & $3.46(0.65-18.39)$ \\
\hline$A R$ & 2 & $98.28(5.22-1849.18)$ \\
\hline $\mathrm{CDH} 13$ & 2 & $10.18(1.71-60.56)$ \\
\hline DDIT3 & 2 & $41.35(5.41-316.24)$ \\
\hline$D K K-1$ & 2 & $24.28(3.15-187.26)$ \\
\hline$E D N R B$ & 2 & $32.57(3.92-270.80)$ \\
\hline$F A N C F$ & 2 & 7.09 (3.71-13.53) \\
\hline$G R A F$ & 2 & $64.36(8.67-477.53)$ \\
\hline$H A G E$ & 2 & 12.23 (1.66-90.39) \\
\hline hPER3 & 2 & $85.56(10.95-668.76)$ \\
\hline $\operatorname{miR}-34 B$ & 2 & $74.99(9.65-582.64)$ \\
\hline$R A G E-1$ & 2 & $38.88(5.25-287.87)$ \\
\hline RUNX3 & 2 & $11.91(1.45-97.86)$ \\
\hline SFRPI & 2 & $23.65(3.05-183.57)$ \\
\hline SHPl & 2 & $11.05(1.41-86.28)$ \\
\hline WIF1 & 2 & $14.15(1.78-112.81)$ \\
\hline$A K A P 12$ & 1 & $34.44(1.85-640.43)$ \\
\hline $\mathrm{CDH1}$ & 1 & $33.00(1.78-610.61)$ \\
\hline$C E B P Z$ & 1 & $35.84(2.12-604.80)$ \\
\hline DRD4 & 1 & $27.26(1.44-516.59)$ \\
\hline E-cad & 1 & $33.00(1.78-610.61)$ \\
\hline$J U N B$ & 1 & $55.00(1.86-1622.60)$ \\
\hline MT3 & 1 & $5.76(1.17-28.24)$ \\
\hline PLCD1 & 1 & $39.38(2.21-702.41)$ \\
\hline PRAME & 1 & $42.49(2.45-737.45)$ \\
\hline$P R D X 2$ & 1 & $36.82(2.14-633.67)$ \\
\hline$R I L$ & 1 & $197.19(11.04-3523.69)$ \\
\hline SOCS-1 & 1 & $34.26(1.79-654.46)$ \\
\hline WNT5A & 1 & $121.51(7.08-2085.83)$ \\
\hline$W W O X$ & 1 & $41.70(2.43-715.18)$ \\
\hline$D L C-1$ & 1 & $17.52(0.90-342.83)$ \\
\hline p53 & 1 & $19.46(0.92-411.20)$ \\
\hline PDLIM4 & 1 & $14.23(0.81-249.59)$ \\
\hline
\end{tabular}

Table I. Continued.

\begin{tabular}{lcc}
\hline Gene & No. of studies & Overall OR $(95 \% \mathrm{CI})$ \\
\hline PTEN & 1 & $16.03(0.83-308.79)$ \\
SALL4 & 1 & $10.67(0.59-192.94)$
\end{tabular}

OR, odds ratio; CI, confidence interval.

Meta-analysis of the association between p73 methylation and leukemia. The meta-analysis of p73 methylation included 7 case-control studies (Fig. 6). The results revealed that hypermethylation of $p 73$ was associated with an increased risk of leukemia $(\mathrm{P}<0.00001 ; \mathrm{OR}=17.07$; 95\% CI: 6.20-47.02). In addition, the results showed that $p 73$ methylation was a risk factor for AML $(\mathrm{P}=0.002 ; \mathrm{OR}=20.83 ; 95 \% \mathrm{CI}$ : 3.01-143.95) and ALL $(\mathrm{P}<0.00001 ; \mathrm{OR}=15.92 ; 95 \% \mathrm{CI}: 4.87-52.07)$, while there was no significant difference between the two subtypes.

Subgroup meta-analysis of gene methylation and leukemia by ethnicity. Based on our previous study (18), a further subgroup meta-analysis by ethnicity was performed for $C D K N 2 A$ and $C D K N 2 B$ methylation. Hypermethylation of $C D K N 2 A$ and $C D K N 2 B$ was associated with an increased risk of leukemia in Chinese populations $(\mathrm{P}<0.00001)$, while only $C D K N 2 B$ was associated with leukemia in Europeans $(\mathrm{P}=0.007)$ (Figs. 7 and 8). Of note, there was a significant difference between European and Chinese populations regarding the association of $C D K N 2 A$ and $C D K N 2 B$ methylation with leukemia $(\mathrm{P}<0.00001$ and $\mathrm{P}=0.02$, respectively).

\section{Discussion}

In the present study, eligible studies were retrieved from the NCBI PubMed and Wanfang literature databases and a systematic meta-analysis was performed to investigate the association between the methylation status of 53 genes and leukemia, with the aim of providing evidence regarding the role of gene methylation in the pathogenesis of leukemia, particularly in different leukemia subgroups and ethnic groups.

Aberrant gene promoter methylation, occurring in almost every tumor type, is one of several mechanisms of gene inactivation (21). Promoter hypermethylation of tumor suppressor genes often contributes to loss of function and cancer development $(22,23)$. One potential mechanism for hypermethylation-induced silencing is changing the structure of specific binding sites for certain transcriptional regulators (24). Epigenetic silencing of genes by promoter hypermethylation is associated with the loss of tumor suppression, increasing tumor severity and reducing patient survival (25). The present meta-analysis revealed significant changes in the methylation status of the $C D K N 2 A, C D K N 2 B$, ID4, GliPR1,p73 and Wilms' tumor 1 (WT1) genes in the major types of leukemia $(21,23,26-28)$.

Numerous studies revealed that $C D K N 2 A$ and $C D K N 2 B$ methylation is frequent during malignant transformation (29-31). As tumor suppressors, $C D K N 2 A$ and $C D K N 2 B$ generate 3 transcript variants (p16 ${ }^{\mathrm{INK} 4 \mathrm{~A}}, \mathrm{p} 14^{\mathrm{ARF}}$ and $\mathrm{p} 15^{\mathrm{INK} 2 \mathrm{~B}}$ ) 
Table II. List of methylated genes and associated case-control studies.

\begin{tabular}{|c|c|c|c|c|c|c|c|c|}
\hline \multirow[b]{2}{*}{ Authors } & \multirow[b]{2}{*}{ Year } & \multirow[b]{2}{*}{ Gene } & \multirow[b]{2}{*}{ Disease } & \multicolumn{2}{|c|}{ Cases (n) } & \multicolumn{2}{|c|}{ Controls (n) } & \multirow[b]{2}{*}{ Refs. } \\
\hline & & & & Meth & Total & Meth & Total & \\
\hline Hsiao et al & 2008 & $C D K N 2 A$ & ALL & 11 & 13 & 0 & 8 & (31) \\
\hline Hsiao et al & 2008 & $C D K N 2 A$ & CLL & 1 & 1 & 0 & 8 & (31) \\
\hline Hsiao et al & 2008 & $C D K N 2 A$ & AML & 5 & 6 & 0 & 8 & (31) \\
\hline Hsiao et al & 2008 & $C D K N 2 A$ & CML & 1 & 3 & 0 & 8 & (31) \\
\hline Zheng et al & 2004 & $C D K N 2 A$ & ALL & 12 & 20 & 0 & 20 & (32) \\
\hline Xiao et al & 2010 & $C D K N 2 A$ & AML & 7 & 21 & 0 & 16 & (33) \\
\hline Xiao et al & 2010 & $C D K N 2 A$ & ALL & 7 & 17 & 0 & 16 & (33) \\
\hline Xiao et al & 2010 & $C D K N 2 A$ & CML & 1 & 7 & 0 & 16 & (33) \\
\hline Xiao et al & 2010 & $C D K N 2 A$ & CLL & 1 & 6 & 0 & 16 & (33) \\
\hline Yang et al & 2003 & $C D K N 2 A$ & ALL & 5 & 28 & 0 & 20 & (34) \\
\hline Yang et al & 2003 & $C D K N 2 A$ & AML & 9 & 43 & 0 & 20 & (34) \\
\hline Song et al & 2004 & $C D K N 2 A$ & ALL & 5 & 28 & 0 & 20 & (35) \\
\hline Tan et al & 2001 & $C D K N 2 A$ & AML & 14 & 20 & 0 & 20 & (36) \\
\hline Zhu et al & 2005 & $C D K N 2 A$ & ALL & 8 & 19 & 0 & 10 & (37) \\
\hline Zhang et al & 2000 & $C D K N 2 A$ & ALL & 20 & 40 & 0 & 15 & (38) \\
\hline Fan et al & 2007 & $C D K N 2 A$ & AML & 24 & 58 & 0 & 16 & (39) \\
\hline Fan et al & 2007 & $C D K N 2 A$ & ALL & 8 & 24 & 0 & 16 & (39) \\
\hline Jiang et al & 2002 & $C D K N 2 A$ & ALL & 19 & 31 & 0 & 20 & (40) \\
\hline Jiang et al & 2002 & $C D K N 2 A$ & AML & 14 & 18 & 0 & 20 & (40) \\
\hline Meng et al & 2005 & $C D K N 2 A$ & AML & 3 & 26 & 0 & 10 & (41) \\
\hline Meng et al & 2005 & $C D K N 2 A$ & ALL & 2 & 14 & 0 & 10 & (41) \\
\hline Wang et al & 2002 & $C D K N 2 A$ & ALL & 11 & 15 & 0 & 12 & (42) \\
\hline Yin et al & 2002 & $C D K N 2 A$ & ALL & 6 & 15 & 0 & 12 & (43) \\
\hline Chen et al & 2003 & CDKN2A (HapII) & AML & 11 & 31 & 0 & 8 & (44) \\
\hline Chen et al & 2003 & $C D K N 2 A(N r u \mathrm{I})$ & AML & 22 & 31 & 2 & 8 & (44) \\
\hline Chen et al & 2003 & CDKN2A (SacII) & AML & 19 & 31 & 1 & 8 & (44) \\
\hline Lin et al & 2012 & $C D K N 2 B$ & ALL & 17 & 25 & 0 & 10 & (45) \\
\hline Zheng et al & 2004 & $C D K N 2 B$ & ALL & 18 & 26 & 0 & 20 & (32) \\
\hline Chen et al & 2003 & $C D K N 2 B$ & AML & 16 & 31 & 0 & 8 & (44) \\
\hline Tan et al & 2001 & $C D K N 2 B$ & AML & 16 & 20 & 0 & 20 & (36) \\
\hline Zhu et al & 2001 & $C D K N 2 B$ & ALL & 12 & 21 & 0 & 10 & (46) \\
\hline Zhu et al & 2005 & $C D K N 2 B$ & ALL & 7 & 19 & 0 & 10 & (37) \\
\hline Shen et al & 2002 & $C D K N 2 B$ & ALL & 6 & 10 & 0 & 10 & (47) \\
\hline Shen et al & 2002 & $C D K N 2 B$ & AML & 10 & 25 & 0 & 10 & (47) \\
\hline Fan et al & 2001 & $C D K N 2 B$ & CML & 5 & 7 & 0 & 20 & (48) \\
\hline Tong et al & 2004 & $C D K N 2 B$ & AML & 5 & 10 & 0 & 10 & (49) \\
\hline Tong et al & 2004 & $C D K N 2 B$ & ALL & 4 & 10 & 0 & 10 & (49) \\
\hline Tong et al & 2004 & $C D K N 2 B$ & CML & 5 & 14 & 0 & 10 & (49) \\
\hline Guo et al & 2000 & $C D K N 2 B$ & AML & 26 & 31 & 0 & 30 & (50) \\
\hline Qiao et al & 2005 & $C D K N 2 B$ & AML & 34 & 42 & 0 & 14 & (51) \\
\hline Qiao et al & 2005 & $C D K N 2 B$ & ALL & 9 & 14 & 0 & 14 & (51) \\
\hline Chen et al & 2000 & $C D K N 2 B$ & ALL & 5 & 10 & 0 & 10 & (52) \\
\hline Yin et al & 2003 & $C D K N 2 B$ & ALL & 6 & 15 & 0 & 12 & (53) \\
\hline Yin et al & 2003 & $C D K N 2 B$ & AML & 17 & 22 & 0 & 12 & (53) \\
\hline Meng et al & 2005 & $C D K N 2 B$ & AML & 24 & 26 & 0 & 10 & (41) \\
\hline Meng et al & 2005 & $C D K N 2 B$ & ALL & 10 & 14 & 0 & 10 & (41) \\
\hline Wu et al & 2013 & $C D K N 2 B$ & ALL & 14 & 14 & 0 & 14 & (54) \\
\hline Wu et al & 2013 & $C D K N 2 B$ & AML & 6 & 14 & 0 & 14 & (54) \\
\hline Wang et al & 2002 & $C D K N 2 B$ & ALL & 7 & 10 & 0 & 7 & (55) \\
\hline Wang et al & 2002 & $C D K N 2 B$ & AML & 22 & 33 & 0 & 7 & (55) \\
\hline
\end{tabular}


Table II. Continued.

\begin{tabular}{|c|c|c|c|c|c|c|c|c|}
\hline \multirow[b]{2}{*}{ Authors } & \multirow[b]{2}{*}{ Year } & \multirow[b]{2}{*}{ Gene } & \multirow[b]{2}{*}{ Disease } & \multicolumn{2}{|c|}{ Cases (n) } & \multicolumn{2}{|c|}{ Controls (n) } & \multirow[b]{2}{*}{ Refs. } \\
\hline & & & & Meth & Total & Meth & Total & \\
\hline Zhao et al & 2008 & ID4 & AML & 15 & 32 & 0 & 18 & (56) \\
\hline Wang et al & 2010 & ID4 & CML & 6 & 48 & 0 & 10 & (57) \\
\hline Liu et al & 2011 & $I D 4$ & AML & 39 & 46 & 0 & 10 & (58) \\
\hline Jie et al & 2012 & ID4 & AML & 21 & 23 & 1 & 20 & (59) \\
\hline Jie et al & 2012 & $I D 4$ & ALL & 9 & 13 & 1 & 20 & (59) \\
\hline Jie et al & 2012 & $I D 4$ & CML & 9 & 11 & 1 & 20 & (59) \\
\hline Zhao et al & 2005 & ID4 & AML & 21 & 25 & 0 & 49 & (60) \\
\hline Zhao et al & 2005 & ID4 & CML & 2 & 4 & 0 & 49 & (60) \\
\hline Zhao et al & 2005 & $I D 4$ & ALL & 12 & 14 & 0 & 49 & (60) \\
\hline Zhao et al & 2005 & $I D 4$ & CLL & 3 & 3 & 0 & 49 & (60) \\
\hline Xiao et al & 2011 & GLIPR1 & AML & 58 & 70 & 14 & 93 & (61) \\
\hline Xiao et al & 2011 & GLIPRI & CML & 11 & 40 & 14 & 93 & (61) \\
\hline Xiao et al & 2011 & GLIPRI & ALL & 22 & 57 & 14 & 93 & (61) \\
\hline Liang et al & 2009 & GLIPR1 & AML & 44 & 54 & 5 & 35 & (62) \\
\hline Liang et al & 2009 & GLIPR1 & CML & 11 & 40 & 5 & 35 & (62) \\
\hline Liang et al & 2009 & GLIPR1 & ALL & 18 & 48 & 5 & 35 & (62) \\
\hline Jie et al & 2012 & GLIPR1 & AML & 22 & 23 & 4 & 20 & (59) \\
\hline Jie et al & 2012 & GLIPRI & ALL & 5 & 13 & 4 & 20 & (59) \\
\hline Jie et al & 2012 & GLIPRI & CML & 6 & 11 & 4 & 20 & (59) \\
\hline Zhang et al & 2010 & p73 & AML & 1 & 30 & 1 & 123 & (63) \\
\hline Zhang et al & 2010 & $p 73$ & ALL & 10 & 112 & 1 & 123 & (63) \\
\hline Zhang et al & 2012 & $p 73$ & AML & 21 & 58 & 0 & 31 & (64) \\
\hline Wu et al & 2008 & $p 73$ & ALL & 10 & 30 & 0 & 16 & (65) \\
\hline Liu et al & 2005 & $p 73$ & ALL & 10 & 26 & 0 & 18 & (66) \\
\hline $\mathrm{Xu}$ et al & 2005 & $p 73$ & ALL & 12 & 42 & 0 & 10 & (67) \\
\hline Yu et al & 2014 & $p 73$ & ALL & 10 & 32 & 0 & 30 & (68) \\
\hline Xie et al & 2003 & $C T$ & AML & 25 & 31 & 0 & 14 & (69) \\
\hline Xie et al & 2003 & $C T$ & CML & 13 & 45 & 0 & 14 & (69) \\
\hline Tang et al & 2001 & $C T$ & CLL & 1 & 3 & 0 & 30 & (70) \\
\hline Tang et al & 2001 & $C T$ & CML & 8 & 10 & 0 & 30 & (70) \\
\hline Tang et al & 2001 & $C T$ & ALL & 12 & 14 & 0 & 30 & (70) \\
\hline Wang et al & 1998 & $C T$ & CML & 13 & 31 & 0 & 10 & (71) \\
\hline Qian et al & 2010 & $D A P K$ & AML & 82 & 112 & 0 & 15 & (72) \\
\hline Niu et al & 2014 & $D A P K$ & AML & 33 & 102 & 0 & 7 & (73) \\
\hline Niu et al & 2014 & $D A P K$ & ALL & 8 & 17 & 0 & 7 & (73) \\
\hline Zhao et al & 2009 & $D A P K$ & AML & 3 & 60 & 0 & 17 & (74) \\
\hline Zhao et al & 2009 & $D A P K$ & ALL & 16 & 55 & 0 & 17 & (74) \\
\hline Qian J & 2008 & $D A P K$ & CML & 25 & 49 & 0 & 13 & (75) \\
\hline Lin W & 2010 & $P R B$ & CLL & 18 & 27 & 0 & 15 & (76) \\
\hline Wu B & 2008 & $P R B$ & CLL & 5 & 9 & 0 & 5 & (77) \\
\hline Zhang et al & 2003 & $P R B$ & ALL & 6 & 11 & 0 & 10 & (78) \\
\hline Zhang et al & 2003 & $P R B$ & CLL & 6 & 8 & 0 & 10 & (78) \\
\hline Zhang et al & 2003 & $P R B$ & AML & 9 & 15 & 0 & 10 & (78) \\
\hline Zhang et al & 2003 & $P R B$ & CML & 6 & 10 & 0 & 10 & (78) \\
\hline Shi et al & 2011 & SFRP5 & AML & 10 & 99 & 1 & 70 & (79) \\
\hline Wang et al & 2012 & SFRP5 & CML & 3 & 3 & 0 & 6 & (80) \\
\hline Wang et al & 2012 & SFRP5 & AML & 4 & 7 & 0 & 6 & (80) \\
\hline $\mathrm{Xu}$ et al & 2010 & SFRP5 & AML & 6 & 59 & 0 & 20 & (81) \\
\hline $\mathrm{Xu}$ et al & 2010 & SFRP5 & ALL & 9 & 28 & 0 & 20 & (81) \\
\hline Li et al & 2004 & $I G S F 4$ & AML & 16 & 29 & 0 & 8 & (82) \\
\hline
\end{tabular}


Table II. Continued.

\begin{tabular}{|c|c|c|c|c|c|c|c|c|}
\hline \multirow[b]{2}{*}{ Authors } & \multirow[b]{2}{*}{ Year } & \multirow[b]{2}{*}{ Gene } & \multirow[b]{2}{*}{ Disease } & \multicolumn{2}{|c|}{ Cases (n) } & \multicolumn{2}{|c|}{ Controls (n) } & \multirow[b]{2}{*}{ Refs. } \\
\hline & & & & Meth & Total & Meth & Total & \\
\hline Li et al & 2004 & IGSF4 & ALL & 12 & 21 & 0 & 8 & (82) \\
\hline Li et al & 2004 & IGSF4 & CML & 6 & 18 & 0 & 8 & (82) \\
\hline Li et al & 2004 & IGSF4 & CLL & 2 & 7 & 0 & 8 & (82) \\
\hline Zhang et al & 2003 & $P R A$ & ALL & 7 & 11 & 0 & 10 & (78) \\
\hline Zhang et al & 2003 & $P R A$ & CLL & 5 & 8 & 0 & 10 & (78) \\
\hline Zhang et al & 2003 & $P R A$ & AML & 10 & 15 & 0 & 10 & (78) \\
\hline Zhang et al & 2003 & $P R A$ & CML & 7 & 10 & 0 & 10 & (78) \\
\hline Chen et al & 2012 & RASSF $1 A$ & AML & 2 & 24 & 0 & 60 & (83) \\
\hline Chen et al & 2012 & $R A S S F 1 A$ & CML & 1 & 23 & 0 & 60 & (83) \\
\hline Chen et al & 2012 & $R A S S F 1 A$ & ALL & 5 & 19 & 0 & 60 & (83) \\
\hline Chen et al & 2012 & RASSF $1 A$ & CLL & 4 & 20 & 0 & 60 & (83) \\
\hline Song et al & 2011 & SFRP2 & CML & 25 & 38 & 0 & 13 & (84) \\
\hline Shi et al & 2011 & $S F R P 2$ & AML & 27 & 99 & 0 & 70 & (79) \\
\hline $\mathrm{Xu}$ et al & 2010 & $S F R P 2$ & AML & 14 & 59 & 0 & 20 & (81) \\
\hline $\mathrm{Xu}$ et al & 2010 & $S F R P 2$ & ALL & 8 & 28 & 0 & 20 & (81) \\
\hline Dou et al & 2004 & LRP15 & AML & 37 & 53 & 0 & 9 & (85) \\
\hline Dou et al & 2004 & LRP15 & ALL & 15 & 20 & 0 & 9 & (85) \\
\hline Dou et al & 2004 & LRP15 & CLL & 1 & 2 & 0 & 9 & (85) \\
\hline Yao et al & 2010 & RIZ1 & AML & 11 & 37 & 0 & 15 & (86) \\
\hline Cai et al & 2012 & RIZ1 & ALL & 15 & 64 & 0 & 9 & (87) \\
\hline Cai et al & 2012 & RIZI & AML & 12 & 32 & 0 & 9 & (87) \\
\hline Shi et al & 2011 & SFRP4 & AML & 17 & 99 & 0 & 70 & (88) \\
\hline $\mathrm{Xu}$ et al & 2010 & SFRP4 & AML & 4 & 59 & 0 & 20 & (81) \\
\hline $\mathrm{Xu}$ et al & 2010 & SFRP4 & ALL & 7 & 28 & 0 & 20 & (81) \\
\hline Jie et al & 2012 & $W T 1$ & AML & 8 & 23 & 15 & 20 & (59) \\
\hline Jie et al & 2012 & $W T 1$ & ALL & 4 & 13 & 15 & 20 & (59) \\
\hline Jie et al & 2012 & $W T 1$ & CML & 7 & 11 & 15 & 20 & (59) \\
\hline Dou et al & 2009 & $\mathrm{ZO}-1$ & Leukemia & 7 & 10 & 0 & 10 & (89) \\
\hline Wang et al & 2008 & $\mathrm{ZO}-1$ & AML & 32 & 52 & 0 & 40 & (90) \\
\hline Wang et al & 2008 & $Z O-1$ & ALL & 17 & 29 & 0 & 40 & (90) \\
\hline Chim et al & 2005 & $R A R A$ & APL & 25 & 63 & 0 & 8 & (91) \\
\hline Chim et al & 2005 & $R A R A$ & AML & 1 & 50 & 0 & 8 & (91) \\
\hline Chim et al & 2005 & RARA & ALL & 1 & 25 & 0 & 8 & (91) \\
\hline Wang et al & 2007 & $A R$ & ALL & 4 & 4 & 0 & 3 & (92) \\
\hline Wang et al & 2007 & $A R$ & AML & 11 & 11 & 0 & 3 & (92) \\
\hline Wang et al & 2009 & $\mathrm{CDH} 13$ & CML & 4 & 8 & 0 & 5 & (93) \\
\hline Liu et al & 2013 & $\mathrm{CDH} 13$ & AML & 23 & 44 & 1 & 10 & (94) \\
\hline Wang et al & 2009 & DDIT3 & AML & 62 & 133 & 0 & 16 & (95) \\
\hline Wang et al & 2009 & DDIT3 & CML & 39 & 59 & 0 & 16 & (95) \\
\hline Zhu et al & 2012 & $D K K-1$ & ALL & 14 & 34 & 0 & 20 & (96) \\
\hline Zhu et al & 2012 & $D K K-1$ & AML & 10 & 31 & 0 & 20 & (96) \\
\hline Yuan et al & 2010 & $E D N R B$ & AML & 15 & 22 & 0 & 8 & (97) \\
\hline Yuan et al & 2010 & $E D N R B$ & ALL & 11 & 17 & 0 & 8 & (97) \\
\hline Yu et al & 2008 & FANCF & AML & 41 & 58 & 7 & 20 & (98) \\
\hline Deng et al & 2009 & FANCF & AML & 85 & 111 & 11 & 42 & (99) \\
\hline Qian et al & 2010 & $G R A F$ & AML & 87 & 132 & 0 & 20 & (100) \\
\hline Qian et al & 2010 & $G R A F$ & CML & 34 & 61 & 0 & 20 & (100) \\
\hline Chen et al & 2012 & $H A G E$ & AML & 32 & 214 & 0 & 24 & (101) \\
\hline Chen et al & 2012 & $H A G E$ & CML & 22 & 87 & 0 & 24 & (101) \\
\hline Li et al & 2011 & hPER3 & CML & 12 & 29 & 0 & 40 & (102) \\
\hline
\end{tabular}


Table II. Continued.

\begin{tabular}{|c|c|c|c|c|c|c|c|c|}
\hline \multirow[b]{2}{*}{ Authors } & \multirow[b]{2}{*}{ Year } & \multirow[b]{2}{*}{ Gene } & \multirow[b]{2}{*}{ Disease } & \multicolumn{2}{|c|}{ Cases (n) } & \multicolumn{2}{|c|}{ Controls (n) } & \multirow[b]{2}{*}{ Refs } \\
\hline & & & & Meth & Total & Meth & Total & \\
\hline Wang et al & 2011 & hPER3 & AML & 116 & 206 & 0 & 40 & (103) \\
\hline Wang et al & 2013 & $m i R-34 B$ & ALL & 24 & 31 & 0 & 23 & (104) \\
\hline Wang et al & 2013 & $m i R-34 B$ & AML & 8 & 19 & 0 & 23 & (104) \\
\hline Chai et al & 2013 & $R A G E-1$ & AML & 52 & 121 & 0 & 25 & $(105)$ \\
\hline Chai et al & 2013 & $R A G E-1$ & CML & 33 & 76 & 0 & 25 & $(105)$ \\
\hline Lin et al & 2008 & RUNX3 & AML & 7 & 23 & 0 & 10 & (106) \\
\hline Lin et al & 2008 & $R U N X 3$ & ALL & 7 & 17 & 0 & 10 & (106) \\
\hline $\mathrm{Xu}$ et al & 2010 & SFRP1 & AML & 20 & 59 & 0 & 20 & $(81)$ \\
\hline $\mathrm{Xu}$ et al & 2010 & SFRPl & ALL & 11 & 28 & 0 & 20 & $(81)$ \\
\hline Chim et al & 2004 & SHPl & AML & 26 & 50 & 0 & 8 & (107) \\
\hline Chim et al & 2004 & SHPl & ALL & 6 & 25 & 0 & 8 & (107) \\
\hline Wang et al & 2011 & WIF1 & AML & 11 & 34 & 0 & 15 & $(108)$ \\
\hline Wang et al & 2011 & WIF1 & ALL & 6 & 21 & 0 & 15 & $(108)$ \\
\hline Liu et al & 2008 & AKAP12 & ALL & 20 & 32 & 0 & 10 & (109) \\
\hline Gao et al & 2006 & $C D H 1$ & AML & 38 & 55 & 0 & 7 & (110) \\
\hline Yao et al & 2011 & $C E B P Z$ & AML & 62 & 133 & 0 & 20 & (111) \\
\hline Guan et al & 2008 & $D L C-1$ & ALL & 21 & 34 & 0 & 5 & (112) \\
\hline Yu et al & 2000 & DRD4 & AML & 16 & 27 & 0 & 9 & (113) \\
\hline Gao et al & 2006 & $E-c a d$ & AML & 38 & 55 & 0 & 7 & (110) \\
\hline Wang et al & 2009 & $J U N B$ & CML & 7 & 8 & 0 & 5 & (93) \\
\hline Tao et al & 2014 & MT3 & AML & 16 & 41 & 2 & 20 & (114) \\
\hline Zheng et al & 2007 & p53 & ALL & 5 & 11 & 0 & 11 & $(115)$ \\
\hline Li et al & 2013 & PDLIM4 & CML & 13 & 59 & 0 & 24 & (116) \\
\hline Song et al & 2012 & PLCDI & CML & 23 & 41 & 0 & 15 & (117) \\
\hline Yao et al & 2013 & PRAME & CML & 28 & 55 & 0 & 20 & (118) \\
\hline Yan et al & 2012 & $P R D X 2$ & AML & 17 & 55 & 0 & 40 & (119) \\
\hline Yang et al & 2007 & PTEN & ALL & 5 & 22 & 0 & 25 & $(120)$ \\
\hline Du et al & 2013 & $R I L$ & AML & 50 & 60 & 0 & 20 & $(121)$ \\
\hline Jiao et al & 2013 & SALL4 & AML & 9 & 45 & 0 & 20 & $(122)$ \\
\hline Zhuang et al & 2011 & SOCS-1 & AML & 15 & 24 & 0 & 10 & $(123)$ \\
\hline Deng et al & 2011 & WNT5A & Leukemia & 47 & 68 & 0 & 27 & (124) \\
\hline Zhang et al & 2012 & $W W O X$ & AML & 23 & 58 & 0 & 31 & (64) \\
\hline
\end{tabular}

ALL, acute lymphocytic leukemia; CLL, chronic lymphocytic leukemia; AML, acute myelogenous leukemia; CML, chronic myelogenous leukemia; Meth, methylated.

according to differences in the first exons and control the progression of cells from the G1 to the S phase $(29,125)$. The present meta-analysis demonstrated that hypermethylation of $C D K N 2 A$ and $C D K N 2 B$ are risk factors for leukemia. According to the subgroup meta-analysis, hypermethylation of $C D K N 2 A$ was significantly associated with AML, ALL and CLL, but not with CML, while $C D K N 2 B$ hypermethylation was significantly associated with AML, ALL and CML. The lack of association of $C D K N 2 A$ with CML may be attributed to the limited sample included in the the meta-analyses (CML power, 6.4\%; and CLL power, 6.3\%).

The ID4 protein is a member of the dominant-negative basic helix-loop-helix transcription factor family that lacks DNA-binding activity (126) and has a tumor suppressor function. The promoter of ID4 was reported to be consistently methylated to various degrees in CLL and a univariate analysis demonstrated that increased promoter methylation of ID4 was correlated with shortened patient survival (127). Previous studies also reported that ID4 gene promoter hypermethylation was highly correlated with acute leukemia and may reflect the malignant degree of AML $(128,129)$. The results of the present meta-analysis demonstrated that methylation of the ID4 gene was associated with an increased risk of leukemia, particularly CML.

The GliPR1 protein, encoded by the GliPRl gene, has been identified as an epigenetically regulated tumor suppressor in prostate cancer and AML. GliPRl may serve as a marker for monitoring disease activity in AML patients during 


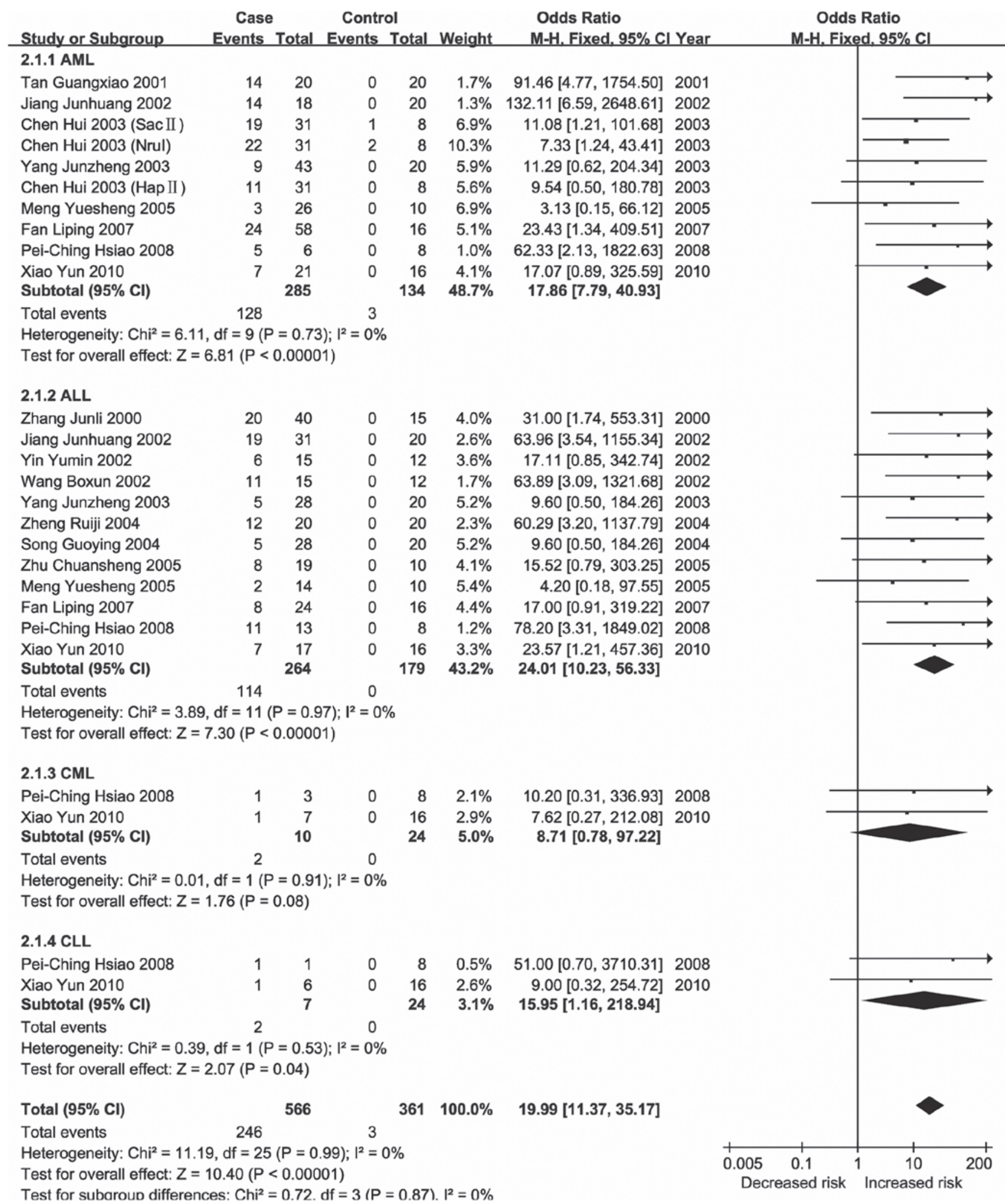

Figure 2. Meta-analyses of aberrantly methylated cyclin-dependent kinase 2A gene in leukemia. ALL, acute lymphocytic leukemia; CLL, chronic lymphocytic leukemia; AML, acute myelogenous leukemia; CML, chronic myelogenous leukemia; M-H, Mantel-Haenszel model; CI, confidence interval; df, degree of freedom.

therapy $(61,130)$. Moreover, GliPRl expression was found to be significantly increased in bone marrow samples of AML patients, while being markedly reduced in ALL, unchanged in myelodysplastic syndrome and marginally decreased in CLL and CML (131). The present meta-analysis identified hypermethylation of the GliPR 1 promoter as a risk factor for leukemia in the Chinese population.

$p 73$, a homologue of the p53 tumor suppressor family, is involved in neurogenesis, sensory pathways, immunity, inflammation and tumorigenesis (132). Furthermore, $p 73$ 


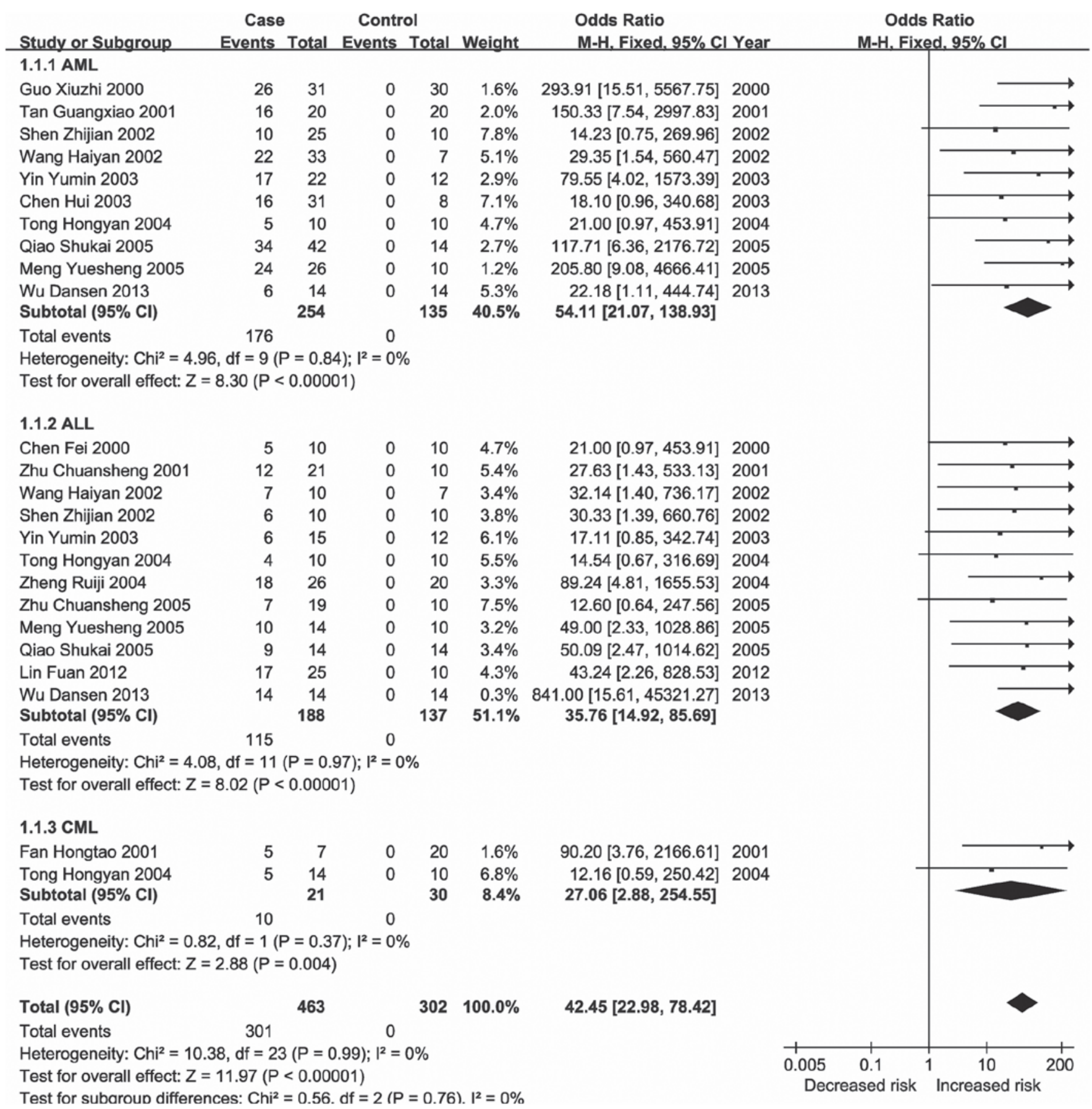

Figure 3. Meta-analyses of aberrantly methylated cyclin-dependent kinase 2B gene in leukemia. ALL, acute lymphocytic leukemia; AML, acute myelogenous leukemia; CML, chronic myelogenous leukemia; M-H, Mantel-Haenszel model; CI, confidence interval; df, degree of freedom.

hypermethylation resulting in its deactivation is frequently observed in malignant lymphoproliferative disorders, particularly ALL (21). In line with these results, the present meta-analysis also identified $p 73$ hypermethylation as a risk factor for leukemia in the Chinese population.

The WT1 gene encodes a zinc finger transcription factor that is an RNA-binding protein with important roles in the development of several organs and tissues. WT1 has been reported to have tumor suppressor as well as oncogenic activity; however. the reasons and mechanisms underlying these opposing functions remain to be fully elucidated (133). The present study demonstrated that WT1 hypermethylation played a protective role against the progression of leukemia.
Previous studies have reported that the risk of hematological malignancies varies significantly among different ethnic groups $(9,13,134,135)$. The present meta-analysis indicated that there was no association between $C D K N 2 A$ methylation and the risk of leukemia $(\mathrm{P}=0.16)$ in Europeans, while a significant association was observed in Chinese populations $(\mathrm{P}<0.00001)$. A significant difference in the association of $C D K N 2 A$ methylation with leukemia was observed between European and Chinese populations $(\mathrm{P}<0.00001)$. This result may provide molecular evidence to guide future individualization of chemotherapy for leukemia, although further research is required to elucidate the precise nature of the ethnic differences in leukemia. 


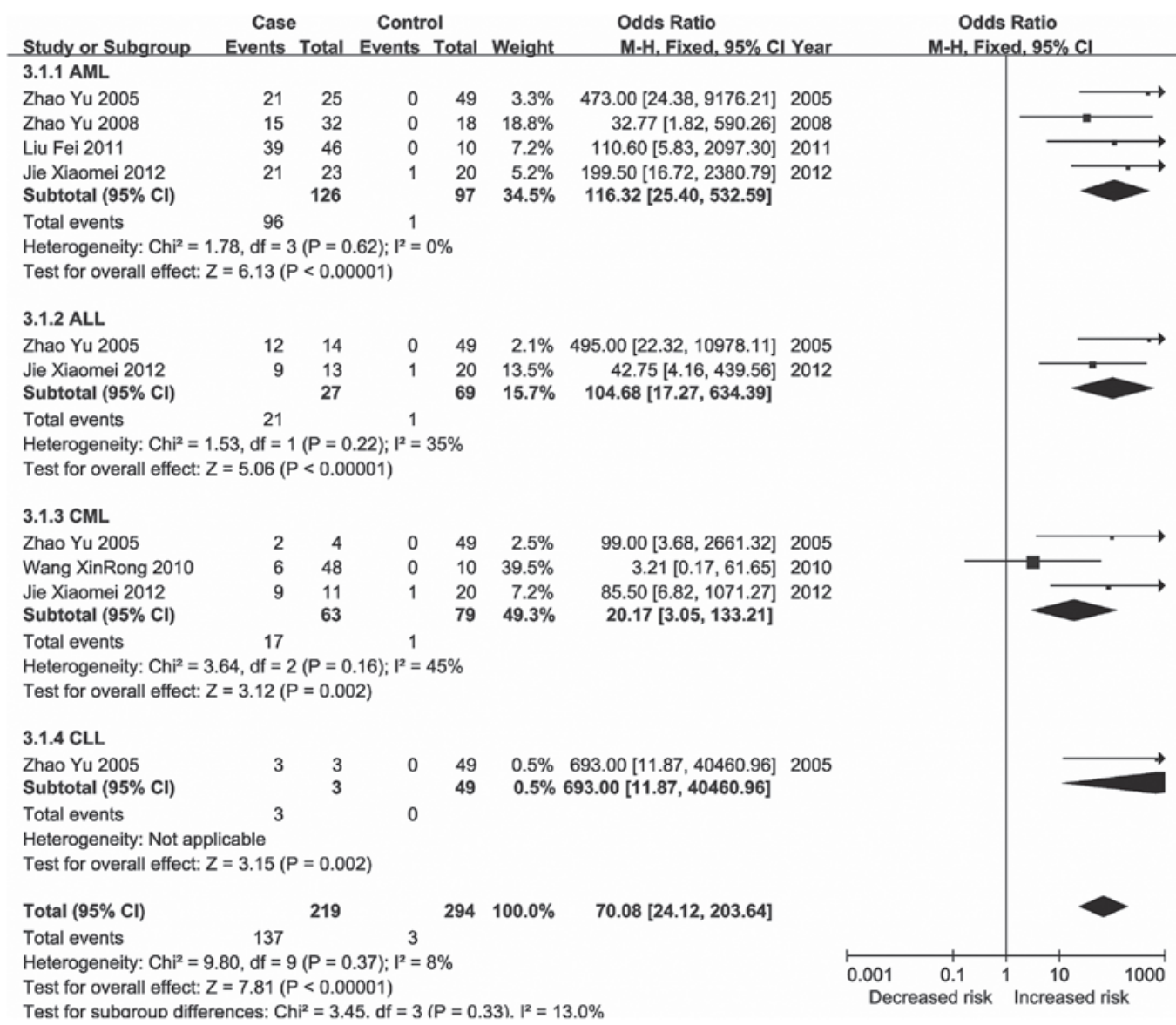

Figure 4. Meta-analyses of aberrantly methylated DNA-binding protein inhibitor-4 gene in leukemia. ALL, acute lymphocytic leukemia; CLL, chronic lymphocytic leukemia; AML, acute myelogenous leukemia; CML, chronic myelogenous leukemia; M-H, Mantel-Haenszel model; CI, confidence interval; df, degree of freedom.

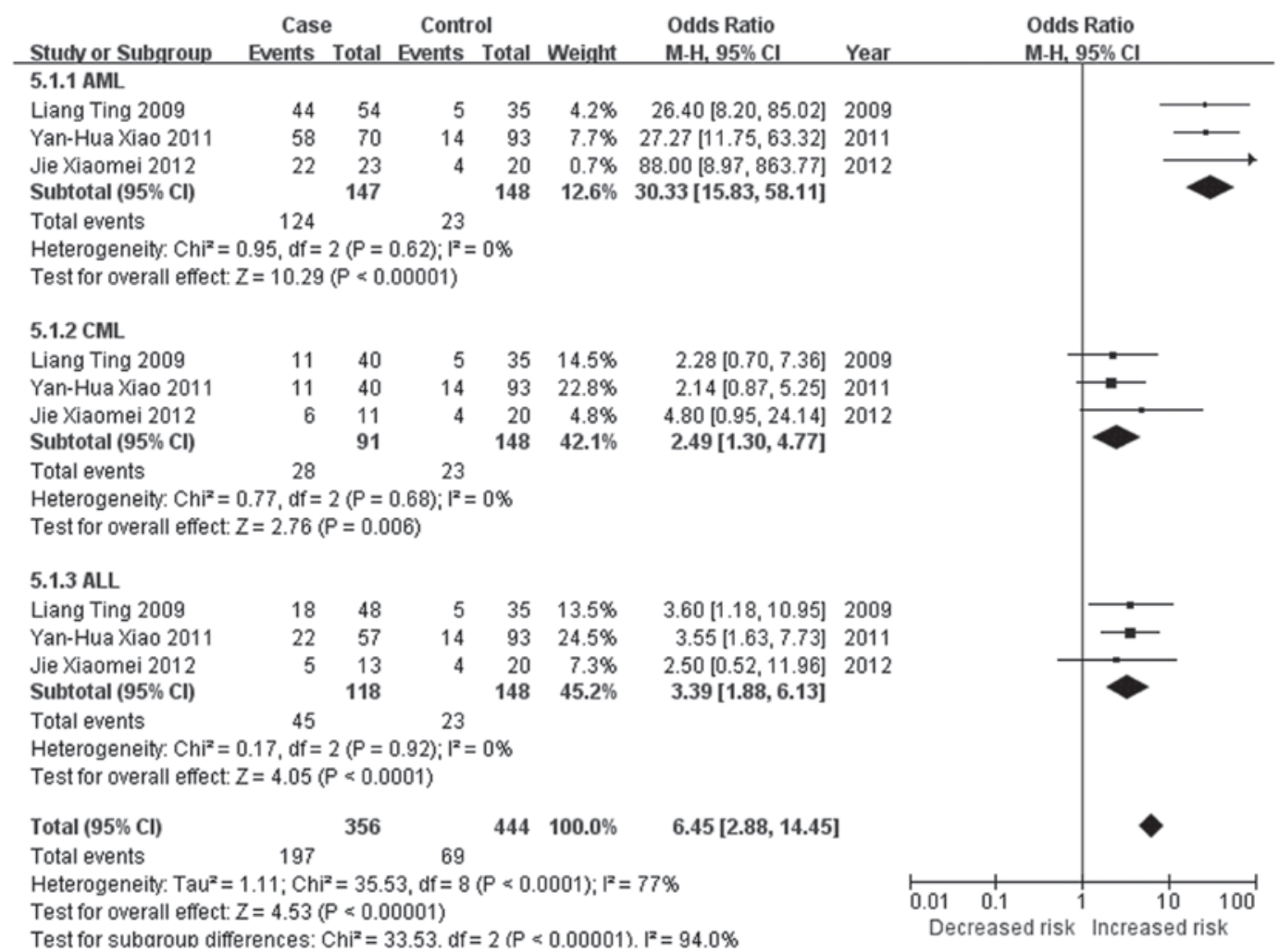

Figure 5. Meta-analyses of aberrantly methylated glioma pathogenesis-related protein 1 gene in leukemia. ALL, acute lymphocytic leukemia; AML, acute myelogenous leukemia; CML, chronic myelogenous leukemia; M-H, Mantel-Haenszel model; CI, confidence interval; df, degree of freedom. 


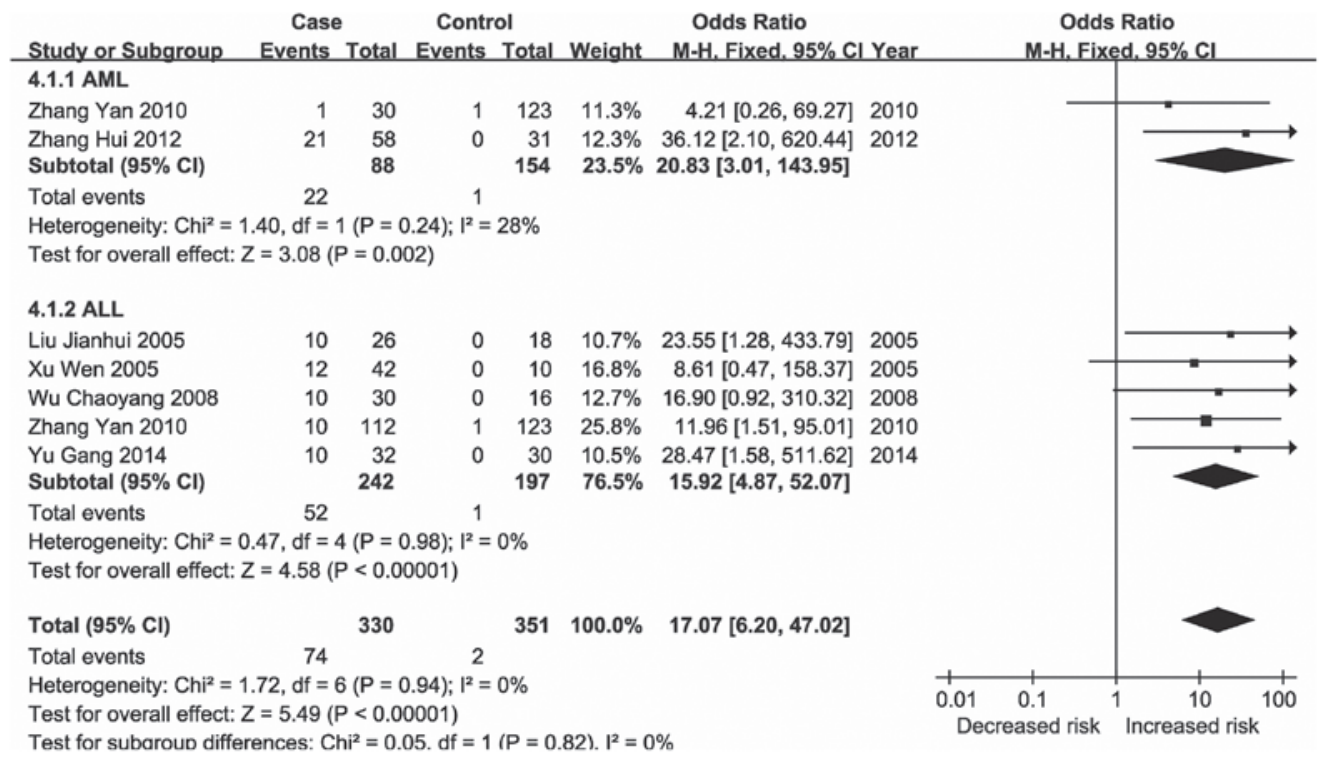

Figure 6. Meta-analyses of aberrantly methylated $p 73$ in leukemia. ALL, acute lymphocytic leukemia; AML, acute myelogenous leukemia; M-H, Mantel-Haenszel model; CI, confidence interval; df, degree of freedom.

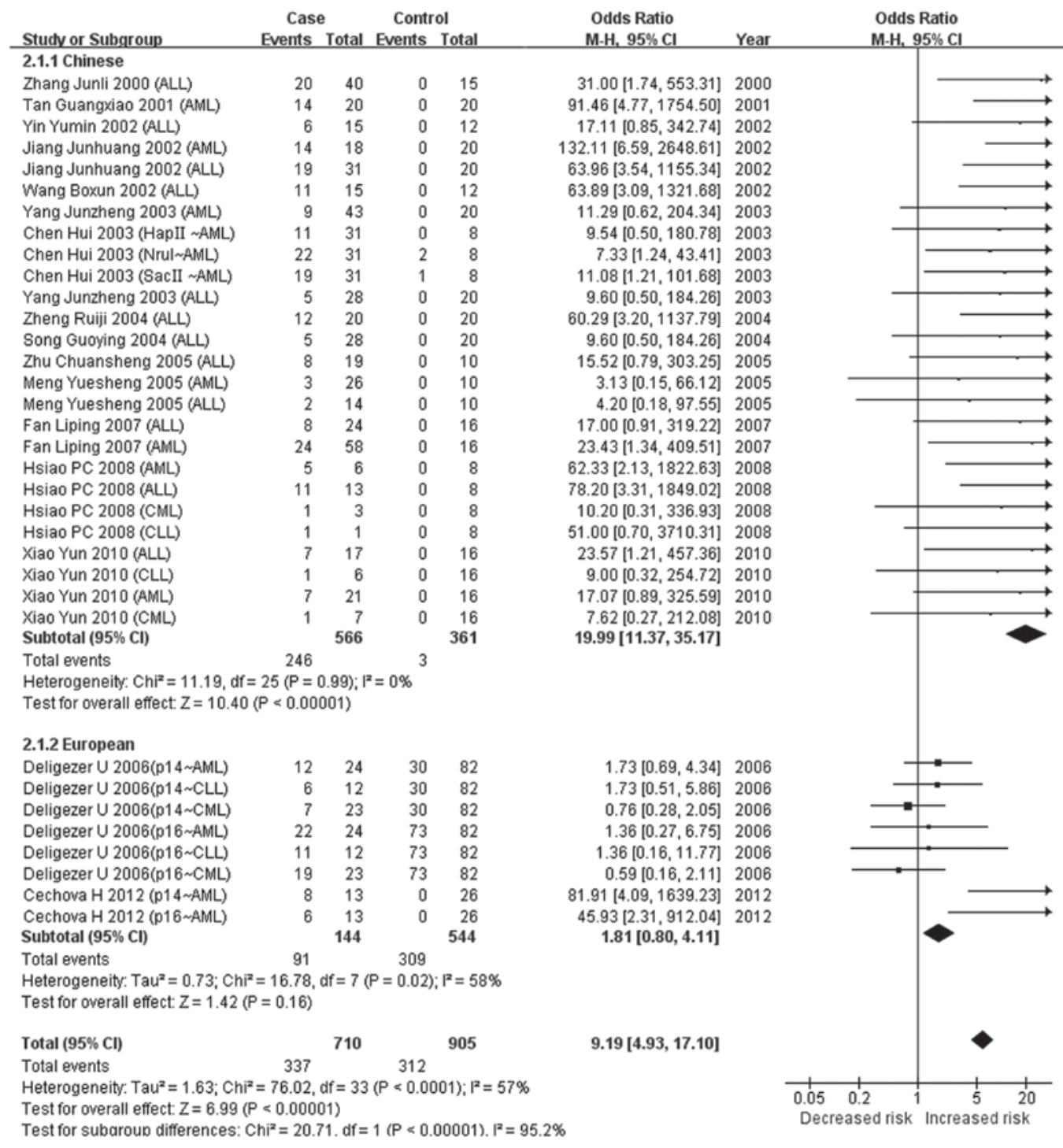

Figure 7. Meta-analyses of aberrantly methylated $C D K N 2 A$ in Asian and European populations. ALL, acute lymphocytic leukemia; CLL, chronic lymphocytic leukemia; AML, acute myelogenous leukemia; CML, chronic myelogenous leukemia; M-H, Mantel-Haenszel model; CI, confidence interval; df, degree of freedom. 


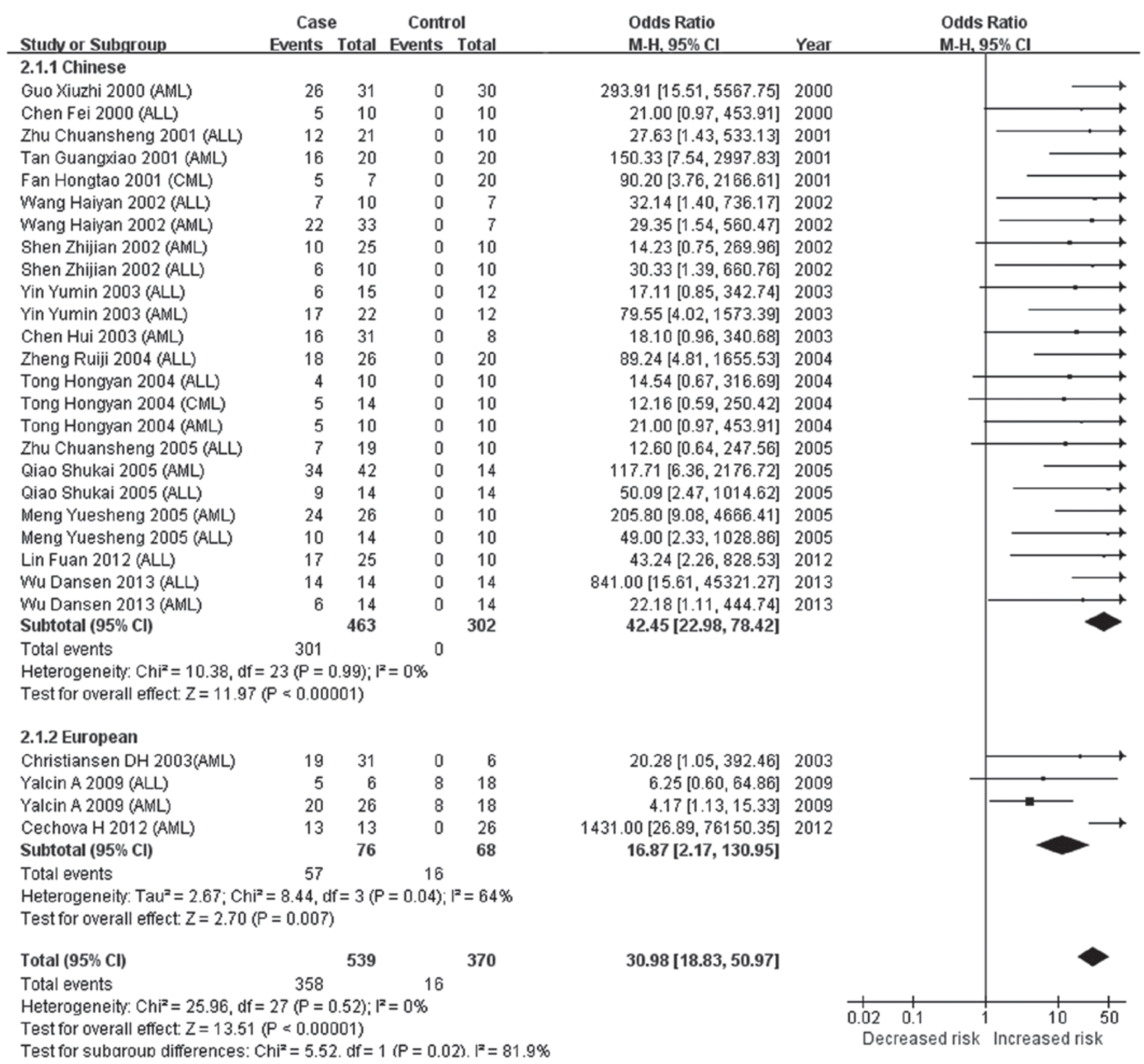

Figure 8. Meta-analyses of aberrantly methylated cyclin-dependent kinase 2B gene in Chinese and European populations. ALL, acute lymphocytic leukemia; AML, acute myelogenous leukemia; CML, chronic myelogenous leukemia; M-H, Mantel-Haenszel model; CI, confidence interval; df, degree of freedom.

Of note, the present meta-analysis had certain limitations. First, the numbers of the studies regarding each gene and leukemia subtype were uneven. For certain leukemia subtypes, only a few studies on certain genes were available. The lack of association of the methylation status of certain genes with several leukemia subtypes may have been due to a lack of statistical power of the respective studies, so that the negative results must be interpreted with caution. Furthermore, a language bias was present, as only studies written in Chinese and English were included.

In conclusion, the present meta-analysis revealed that aberrant DNA methylation of the promoters of 47 genes was associated with leukemia. Further subgroup meta-analysis revealed 5 hypermethylated genes $(C D K N 2 A, C D K N 2 B, I D 4$, GliPRI and $p 73)$ in various leukemia subtypes. In addition, a difference in the association of $C D K N 2 A$ and $C D K N 2 B$ hypermethylation with leukemia was identified between Chinese and European populations. The results of the present study may enhance the current understanding of the association of DNA methylation with leukemia in the Chinese population.

\section{Acknowledgements}

The present study was supported by grants from National Natural Science Foundation of China (nos. 31100919 and 81371469), Natural Science Foundation of Zhejiang Province (no. LR13H020003), K.C. Wong Magna Fund at Ningbo University and Ningbo Social Development Research Projects (nos. 2010C50019 and 2012C50032). 


\section{References}

1. Davis AS, Viera AJ and Mead MD: Leukemia: An overview for primary care. Am Fam Physician 89: 731-738, 2014.

2. Zhong S, Chen Z, Yu X, Chen W, Lv M, Ma T and Zhao J: Tea consumption and leukemia risk: A meta-analysis. Tumour Biol 35: 5205-5212, 2014

3. Greim H, Kaden DA, Larson RA, et al: The bone marrow niche, stem cells, and leukemia: Impact of drugs, chemicals, and the environment. Ann NY Acad Sci 1310: 7-31, 2014.

4. Laurier D, Grosche B, Auvinen A, et al: Childhood leukaemia risks: From unexplained findings near nuclear installations to recommendations for future research. J Radiol Prot 34: R53-R68 2014.

5. Malinge S, Chlon T, Doré LC, et al: Development of acute megakaryoblastic leukemia in Down syndrome is associated with sequential epigenetic changes. Blood 122: e33-e43, 2013.

6. Wakeford R: The risk of childhood leukaemia following exposure to ionising radiation - a review. J Radiol Prot 33: 1-25, 2013.

7. Woo JS, Alberti MO and Tirado CA: Childhood B-acute lymphoblastic leukemia: A genetic update. Exp Hematol Oncol 3: 16 , 2014.

8. Bhatia S: Disparities in cancer outcomes: Lessons learned from children with cancer. Pediatr Blood Cancer 56: 994-1002, 2011.

9. Carranza C, Granados L, Morales O, et al: Frequency of the ETV6-RUNX1, BCR-ABL1, TCF3-PBX1, and MLL-AFF1 fusion genes in Guatemalan pediatric acute lymphoblastic leukemia patients and their ethnic associations. Cancer Genet 206: 227-232, 2013.

10. Goggins WB and Lo FF: Racial and ethnic disparities in surviva of US children with acute lymphoblastic leukemia: Evidence from the SEER database 1988-2008. Cancer Causes Control 23: 737-743, 2012.

11. Kennedy AE, Kamdar KY, Lupo PJ, et al: Genetic markers in a multi ethnic sample for childhood acute lymphoblastic leukemia risk. Leuk Lymphoma 56: 169-174, 2014.

12. Savage SA: Genomic clues to ethnic differences in ALL. Blood 123: 2440-2442, 2014

13. Lim JY, Bhatia S, Robison LL and Yang JJ: Genomics of racial and ethnic disparities in childhood acute lymphoblastic leukemia. Cancer 120: 955-962, 2014.

14. Adkins RM, Krushkal J, Tylavsky FA and Thomas F: Racial differences in gene-specific DNA methylation levels are present at birth. Birth Defects Res A Clin Mol Teratol 91: 728-736, 2011

15. Xia YY, Ding YB, Liu XQ, et al: Racial/ethnic disparities in human DNA methylation. Biochim Biophys Acta 1846: 258-262. 2014.

16. Baylin SB and Jones PA: A decade of exploring the cancer epigenome - biological and translational implications. Nat Rev Cancer 11: 726-734, 2011

17. Plass C, Pfister SM, Lindroth AM, et al: Mutations in regulators of the epigenome and their connections to global chromatin patterns in cancer. Nat Rev Genet 14: 765-780, 2013.

18. Jiang D, Hong Q, Shen Y, et al: The diagnostic value of DNA methylation in leukemia: A systematic review and meta-analysis. PLoS One 9: e96822, 2014

19. Higgins JP, Thompson SG, Deeks JJ and Altman DG: Measuring inconsistency in meta-analyses. BMJ 327: 557-560, 2003.

20. Bax L, Ikeda N, Fukui N, Yaju Y, Tsuruta $\mathrm{H}$ and Moons KG: More than numbers: The power of graphs in meta-analysis. Am J Epidemiol 169: 249-255, 2009.

21. Pluta A, Nyman U, Joseph B, Robak T, Zhivotovsky B and Smolewski P: The role of p73 in hematological malignancies. Leukemia 20: 757-766, 2006

22. Cahill N and Rosenquist R: Uncovering the DNA methylome in chronic lymphocytic leukemia. Epigenetics 8: 138-148, 2013.

23. Esteller $\mathrm{M}: \mathrm{CpG}$ island hypermethylation and tumor suppressor genes: A booming present, a brighter future. Oncogene 21: 5427-5440, 2002.

24. Zhao Y, Guo J, Zhang X, et al: Downregulation of p21 in myelodysplastic syndrome is associated with p73 promoter hypermethylation and indicates poor prognosis. Am J Clin Pathol 140: 819-827, 2013.

25. Volodko N, Gordon M, Salla M, Ghazaleh HA and Baksh S: RASSF tumor suppressor gene family: Biological functions and regulation. FEBS Lett 588: 2671-2684, 2014.

26. Benezra R, Davis RL, Lockshon D, Turner DL and Weintraub H: The protein Id: A negative regulator of helix-loop-helix DNA binding proteins. Cell 61: 49-59, 1990.
27. Bodoor K, Haddad Y, Alkhateeb A, et al: DNA hypermethylation of cell cycle (p15 and p16) and apoptotic (p14, p53, DAPK and TMS1) genes in peripheral blood of leukemia patients. Asian Pac J Cancer Prev 15: 75-84, 2014.

28. Chmelařová M, Křepinská E, Spaček J, Laco J, Nekvindová J and Palička V: Methylation analysis of tumour suppressor genes in ovarian cancer using MS-MLPA. Folia Biol (Praha) 58: 246-250, 2012.

29. Cechova H, Lassuthova P, Novakova L, et al: Monitoring of methylation changes in 9p21 region in patients with myelodysplastic syndromes and acute myeloid leukemia. Neoplasma 59: 168-174, 2012.

30. Deligezer U, Erten N, Akisik EE and Dalay N: Methylation of the INK4A/ARF locus in blood mononuclear cells. Ann Hematol 85 102-107, 2006.

31. Hsiao PC, Liu MC, Chen LM, et al: Promoter methylation of p16 and EDNRB gene in leukemia patients in Taiwan. Chin J Physiol 51: 27-31, 2008

32. Zheng R, Shen S, Shen J and Ma X: Studies on p16, p15, p18 and p19 methylation of INK4 series cancer inhibitive gene in leukemia. J Fujian Med Univ 38: 257-260, 2004.

33. Xiao Y, Liu Y, Jiang L and Ran L: Study on the deletion point mutation and promoter methylation analysis of p16 in leukemia. Guizhou Med J 34: 784-787, 2010.

34. Yang J, Li X, Sun L, Song G, Chen H and Liu Y: Expression of p16 gene in adult acute leukemia. J Zhengzhou Univ (Med Sci Ed.) 38: 33-36, 2003 .

35. Song G, Yang J, Sun H, Liu C, Chen H and Li X: Deletion and methylation of p16 gene in an adult acute leukemia. Henan J Oncol 17: 229-231, 2004.

36. Tan $\mathrm{G}$ and Guo X: Methods of $\mathrm{CpG}$ islands methylation detection. J Jinan Univ 22: 24-26, 2001.

37. Zhu C, Xu W, Liu D, Wang Y and Bi K: Study of the methylation of p15 and p16 gene in acute leukemia. Shandong Med J 45: 4-5, 2005.

38. Zhang J, Wu J and Tan Y: The relationship between P16 gene inactivation and expression of mRNA in ALL. J Wenzhou Med Coll 30: 5-7, 2000

39. Fan L, Shen J, Ye B, et al: Detection of p16 gene methylation status in adult patients with acute leukemia by using n-MSP. J Exp Hematol 15: 258-261, 2007.

40. Jiang J, Shen J, Yan J, et al: The value of p15 gene methylation in minimal residual disease of childhood acute leukemia by MSP. China Child Blood 7: 1-3, 2002.

41. Meng Y, Yu H, Guo C, et al: Quantitative analysis of p15 and p16 gene methylation and mRNA expression levels in acute leukemia patients. Chin J Hematol 26: 434-435, 2005.

42. Wang B: The clinical significance of p16 gene methylation in leukemia and other hematologic malignancies. Master's degree dissertation, China Medical University, 2002.

43. Yin Y: The clinical significance of p15 gene methylation in leukemia and other hematologic malignancies. Master's degree dissertation, China Medical University, 2002.

44. Chen H, Sun H, Liu C, Liu Y, Song G and Li X: Methylation of p16/p15 exon 2 in adult acute myeloid leukemia. J Zhengzhou Univ (Med Sci Ed.) 38: 722-724, 2003.

45. Lin F, Ye B, Shen J, et al: Detection of p15 methylation and deletion status in acute lymphoblastic leukemia using hn-MSP. J Leuk Lymphoma 21: 208-212, 2012.

46. Zhu C, Bi K and Liu X: Homozygous deletion and methylation of P15 gene in acute laukemia. Shandong Med J Shandong Yiyao 41: 10-11, 2001.

47. Shen Z, Zhang J, Yu K and Jiang S: Evaluation of methylationspecific polymerase chain reaction and its potential clinical value in detecting minimal residual disease of adult acute leukemia with P15. J Wenzhou Med Coll 32: 230-232, 2002.

48. Fan H, Guo X, Tan G, Wu Q, Zhou T and Guo Q: Study on methylation of p15 INK4B in myelodysplastic syndrome. Chin J Pathophysiol 17: 349-352, 2001

49. Tong $\mathrm{H}$ and Lin $\mathrm{M}$ : Methylation of p15INK4B gene and its mechanism in patients with myelodysplastic syndrome and leukemia. Chin J Pathophysiol 20: 2257-2260, 2004.

50. Guo X, Fan H, Wu Q, et al: Study on methylation of p15INK4B gene in acute myeloid leukemia and chronic myeloid leukemia. J Exp Hematol 8: 257-260, 2000,

51. Qiao S, Xu S, Guo X and Wang Y: Clinical significance of the expression of DNA methyltransferase genes (DNMT) in acute leukemia patients. J Exp Hematol 13: 260-265, 2005.

52. Chen F and Zeng X: Detection of p15 gene hypermethylation in acute leukemia by MSP. Chin J Hematol 21: 250, 2000. 
53. Yin Y, Li Y and Liu D: The clinical significance of p15 gene methylation in leukemia and other hematologic malignancies. Chin J Hematol 24: 383-384, 2003.

54. Wu D: p15INK4b gene DNA methylation and histone modification mechanism of leukemia cell mediated by p15-piRNAs. PhD dissertation, Fujian Medical University, 2013.

55. Wang H: The study of p15 gene methylation in malignancies. Master's degree dissertation, Qingdao University, 2002.

56. Zhao Y, Wang QS, Li HH, et al: [Significance of id4 promoter methylation in monitoring AML patients with completely remission]. J Exp Hematol 16: 476-478, 2008.

57. Wang X, Kang H, Cen J, Li Y, Wang L and Yu L: Methylation Status of id4 gene promoter in patients with chronic myeloid leukemia. J Exp Hematol 18: 1402-1404, 2010.

58. Liu F, Xu R, Cui X, Zhang X and Wang Y: ID4 promoter methylation in acute myeloid leukemia. J Exp Hematol 19: 582-584, 2011.

59. Jie X: WT1, ID4, GLIPR1 aberrant gene promoter methylation and mRNA expression in leukemia patients. Master's degree dissertation, Guangdong Medical College, 2012.

60. Zhao Y: The significance of Id4 gene methylation in hematological malignancies. PhD dissertation, PLA Postgraduate Medical School, 2005.

61. Xiao YH, Li XH, Tan T, et al: Identification of GLIPR1 tumor suppressor as methylation-silenced gene in acute myeloid leukemia by microarray analysis. J Cancer Res Clin Oncol 137: 1831-1840, 2011.

62. Liang T, Tan T, Xiao Y, et al: Methylation and expression of glioma pathogenesis-related protein 1 gene in acute myeloid leukemia. J Cent South Univ (Med Sci Ed.) 34: 388-394, 2009.

63. Zhang Y, Gao Y, Wang X, et al: The relationship between $\mathrm{p} 73$ gene methylation and childhood acute leukemia. In: Proceedings of the Ninth National Conference on Environmental and Occupational Medicine Graduate, Shanghai, China: 254-256, 2010.

64. Zhang H, Mo W and Yang Z: The expression of WWOX and p73 in acute myeloid leukemia. Guangdong Med J 33: 1567-1570, 2012.

65. Wu C, Jin J, Ji Y, et al: Abnormal expression and significance of p73 gene in adult acute lymphoblastic leukemia. Jiangsu Med J 34: 339-341, 2008

66. Liu J, Jiang Y, Xu W and Liang X: Alterations of p53 and p73 genes in lympho-plasmacytic diseases. Pract Clin Med 6: 9-13, 2005.

67. Xu W: Study on methylation of the p73 gene in acute lymphoblastic leukemia and its clinical significances. Chin J Pract Int Med 25: 905-906, 2005.

68. Yu G: Abnormal expression of p73 gene in adult acute lymphoblastic leukemia. Med Innov Chin 2: 121-122, 2014.

69. Xie X, Chen Z, Zhang X and Huang M: Detecting methylation of the calcitonin gene in monitoring treatment and disease evolution for myelogenous leukemia. Chin J Cancer 22: 616-619, 2003.

70. Tang Y, Deng C, Du Q and Li G: Study on hypermethylation of the calcitonin gene in malignant hematological disorders. J West Chin Univ Med Sci 32: 86-88, 2001.

71. Wang S, Hong $\mathrm{W}$ and Peng A: The study of CT gene methylation in chronic myeloid leukemia. Chin J Hematol 19: 32, 1998.

72. Qian J, Yao D, Lin J, et al: Alteration of methylation status of death-associated protein kinase (dapk) gene promoter in patients with acute myeloid leukemia. J Exp Hematol 18: 1390-1394, 2010.

73. Niu Y, Wang P, Wang Y, Wang Y, Cai D and Li Y: Expression of death-associated protein kinase gene and methylation status of promoter region in acute leukimia. J Exp Hematol 22: 30-34, 2014.

74. Zhao W: DAPK gene promoter methylation, expression and biological function in acute leukemia. PhD dissertation, Southern Medical University, 2009.

75. Qian J, Wang YL, Lin J, Yao DM, Xu WR and Wu CY: Aberrant methylation of the death-associated protein kinase 1 (DAPK1) $\mathrm{CpG}$ island in chronic myeloid leukemia. Eur J Haematol 82: 119-123, 2009.

76. Lin W, Ye Z and Tan J: Gene methylation abnormalities in chronic lymphocytic leukemia patients by PCR. J Qiqihar Med Coll 31: 2376-2377, 2010.

77. Wu B, Chen E and Zhu B: Detecting the aberrant methylation of progesterone receptor B gene in human chronic lymphocytic leukemia by MSP. Chin J Hemorheol 18: 140-141, 2008.

78. Zhang X: The experimental study of PR promoter $\mathrm{CpG}$ island methylation in leukemia. Master's degree dissertation, Third Military Medical University, 2003.

79. Shi X, Chang N, Fan Y, et al: Detection and clinical significance of SFRP2 promoter methylation status in patients with acute myeloid leukemia. Chin J Clin, Electron Ed. 5: 1962-1966, 2011.
80. Wang H, Zhang G, Yao K, et al: The gene methylation and protein expression of SFRP5 in leukemic cells. J China Med Univ 41: 2012

81. Xu C, Shen J, Shen S, Fu H, Zhu Y and Chen L: The significance of methylation status of secreted frizzled-related protein gene promoter in acute leukemia. Chin J Intern Med 49: 769-771, 2010.

82. Li M: A preliminary study of inactivation of IGSF4 gene methylation in leukemia cell. PhD dissertation, PLA Postgraduate Medical School, 2004.

83. Chen W, Huang C, Zhan X, et al: RASSF1A gene methylation and its clinical significance in leukemia patients. Lab Med 27: 554-556, 2012

84. Song J, Li Y, Yang Z, Yang L and Chen J: Hypermethylation in SFRP2 promoter in human leukemia K 562 cells and bone marrow specimen with chronic myeloid leukemia. Acad J Third Med Coll PLA 33: 2583-2586, 2011

85. Dou L: The study of LRP15 gene methylation in blood diseases. Master's degree dissertation, PLA Postgraduate Medical School, 2004.

86. Yao C, Fang J, Gao L, Ding J, Shu G and Yu W: Study on the relationship between the RIZ1 gene expression and methylation status of the gene promoter in acute myeloid leukemia. J Southeast Univ (Med Sci Ed.) 29: 119-122, 2010.

87. Cai F: RIZ1 gene expression and promoter methylation status in acute childhood leukemia. PhD dissertation, Huazhong University Science and Technology, 2012.

88. Shi X, Fan Y, Zhou C, et al: Study of abnormal methylation in the promoter region of SFRP4 gene in acute myeloid leukemia. Chin J Clin, Electron Ed. 5: 990-995, 2011.

89. Dou LP, Liu JH, Wang C, et al: [Study on the involvement of ZO-1 gene in leukemogenesis]. Chin J Hematol 30: 473-476, 2009.

90. Wang C, Wang G, Tan Y, Li W, Liu C and Yu L: The methylation pattern and clinical significance of Zonula occludens-1 gene promoter in acute leukemia. Chin J Intern Med 47: 111-113, 2008.

91. Chim CS, Wong SY, Pang A, et al: Aberrant promoter methylation of the retinoic acid receptor alpha gene in acute promyelocytic leukemia. Leukemia 19: 2241-2246, 2005.

92. Wang G, Liu L, Chen J, et al: Promoter methylation profile of androgen receptor gene in leukemic cells. J Third Mil Med Univ 29: 932-934, 2007.

93. Wang X, Li J, Fu B, Guo L, Zhang J and Huang S: Methylation Status of JunB and CDH13 gene promoter in CD34 CD38 chronic myelogenous leukemia cells. J Exp Hematol 17: 1405-1408, 2009.

94. Liu J: CDH13 gene promoter methylation and expression in acute myeloid leukemia. Master's degree dissertation, Guangzhou Medical University, 2013.

95. Wang Y: The study of DDIT3 gene promoter methylation in hematological tumors. Master's degree dissertation, Jiangsu University, 2009.

96.Zhu X, Zhu C, Wan Y, et al: Analysis of methylation of the Dickkopf1 (DKK-1) gene in acute leukemia. J Shandong Univ (Health Sci) 50: 84-87, 2012

97. Yuan X: The study of EDNRB gene promoter methylation in acute leukemia. Master's degree dissertation, Zhengzhou University, 2010.

98. Yu Y: The study of FANCF methylation in patients with acute myeloid leukemia and myelodysplastic syndrome. Master's degree dissertation, China Medical University, 2008.

99. Deng N: The study of FANCF/FANCD2 expression in patients with acute myeloid leukemia and myelodysplastic syndrome. Master's degree dissertation, China Medical University, 2009.

100. Qian Z: The significance of GRAF gene promoter methylation in hematopoietic tumors. Master's degree dissertation, Jiangsu University, 2010.

101. Chen Q: HAGE gene promoter hypomethylation in myeloid tumors. Master's degree dissertation, Jiangsu University, 2012.

102. Li Y, Wang Y, Zhou J, Zhou S, Fang G and Liu X: Analysis and clinical significance of hPer3 promoter methylation status for CML monitoring. Chin J Pathophysiol 27: 2111-2115, 2011.

103. Wang Y, Zhou J, Zhou S, et al: Promoter methylation status of hPer3 gene in AML patients and the in vitro effect of decitabine on the status. Chin J Hematol 32: 317-321, 2011.

104. Wang N: MiR-34b expression, methylation regulation and clinical significance in acute childhood leukemia. Master's degree dissertation, Suzhou University, 2013.

105. Chai H: RAGE-1 gene promoter hypomethylation in myeloid tumors. Master's degree dissertation, Shanxi Medical University, 2013. 
106. Lin D, Fan R and Liu X: Significance of DNA methylation status of runx3 gene promoter region in acute leukemia. J Exp Hematol 16: 263-266, 2008.

107. Chim CS, Wong AS and Kwong YL: Epigenetic dysregulation of the Jak/STAT pathway by frequent aberrant methylation of SHP1 but not SOCS1 in acute leukaemias. Ann Hematol 83: 527-532, 2004.

108. Wang Y, Zhu CS, Bi KH, Xu WW, Dong L and Hou M: [Study of WIF-1 promoter methylation with expressions of $\beta$-catenin in acute leukemia]. Chin Med J 91: 2858-2860, 2011.

109. Liu W, Xu C, Guan M and Zhang Y: Aberrant promoter hypermethylation of AKAP12 gene in acute lymphoblastic leukemia in children. Lab Med 23: 344-348, 2008.

110. Gao F, Li Y, Liu W, et al: Studies on gene expression and the 5 $\mathrm{CpG}$ islands methylation status of E-cadherin in acute myeloid leukemia. Chin J Hematol 27: 25-27, 2006.

111. Yao DM, Qian J, Lin J, et al: Aberrant methylation of CCAAT/enhancer binding protein zeta promoter in acute myeloid leukemia. Leuk Res 35: 957-960, 2011.

112. Guan M, Xu C, Liu W and Chen B: Identification of aberrant methylation of DLC-1 by pyrosequencing in acute lymphoblastic leukemia in children. Chin J Lab Med 31: 389-393, 2008.

113. Yu L, Wang Q, Gu Q, Shi Z and Jin H: Study on aberrant DNA methylation of dopamine D4 receptor gene in acute myeloid leukemia. Acad J Chin PLA Postgrad Med Sch 21: 62-64, 2000.

114. Tao YF, Xu LX, Lu J, et al: Metallothionein III (MT3) is a putative tumor suppressor gene that is frequently inactivated in pediatric acute myeloid leukemia by promoter hypermethylation. J Trans Med 12: 182, 2014.

115. Zheng Y: Abnormal methylation of the promoter region of the p53 gene in acute leukemia. Master's degree dissertation, Sun Yat-sen University, 2007.

116. Li Y, Qian J, Chen Q, et al: Expression pattern and promoter hypermethylation of PDLIM4 gene in patients with chronic myeloid leukemia. J Jiangsu Univ (Med Ed.) 23: 17-21, 2013.

117. Song JJ, Liu Q, Li Y, et al: Epigenetic inactivation of PLCD1 in chronic myeloid leukemia. Int J Mol Med 30: 179-184, 2012.

118. Yao D, Qian J, Lin J, Li Y, Chen Q and Chen X: Alteration of methylation status of PRAME gene promoter in patients with chronic myeloid leukemia. J Pract Med 29: 3495-3498, 2013.

119. Yan W, Hu W and Yang W: Expression and methylation study of PRDX2 gene in acute myeloid leukemia. J China Med Univ 41: 1047-1049, 2012.

120. Yang J, Du W, He Y, Liu J, Zheng J and Huang S: Research on PTEN expression and its promoter methylation in B-ALL patients. J Intern Intens Med 13: 141-143, 2007.

121. Du C, Mao P and Wang Y: The clinical significance of the RIL gene methylation levels in acute myeloid leukemia. China J Mod Med 23: 46-51, 2013.

122. Jiao X, Chen Q, Lin J, et al: SALL4 genes methylation changes and clinical significance in acute myeloid leukemia patients. J Jiangsu Univ (Med Ed.) 23: 532-535, 2013.
123. Zhuang Y, Cheng Y, Wang L, Dou H, Zhu Q and Hu J: The methylation of SOCS-1 gene in adult acute myeloid leukemia. Prog Mod Biomed 18, 3417-3420, 2011.

124. Deng G, Li ZQ, Zhao C, et al: WNT5A expression is regulated by the status of its promoter methylation in leukaemia and can inhibit leukemic cell malignant proliferation. Oncol Rep 25: 367-376, 2011

125. Iacobucci I, Ferrari A, Lonetti A, et al: CDKN2A/B alterations impair prognosis in adult BCR-ABL1-positive acute lymphoblastic leukemia patients. Clin Cancer Res 17: 7413-7423, 2011.

126. $\mathrm{Hu} \mathrm{HB}$ and $\mathrm{Hu} \mathrm{Q}$ : ID4 methylation patterns in childhood $\mathrm{T}$ line and B line lymphocytic leukemia. Chin J Contemp Pediatr 12: 940-942, 2010 (In Chinese).

127. Chen SS, Claus R, Lucas DM, et al: Silencing of the inhibitor of DNA binding protein 4 (ID4) contributes to the pathogenesis of mouse and human CLL. Blood 117: 862-871, 2011.

128. Liu Y, Kang HY, Wang LL, Lu XC, Zhu HL and Yu L: Establishment of methylation-specific quantitative PCR system for ID4 gene in acute leukemia cells and its specificity and sensitivity. J Exp Hematol 22: 269-274, 2014 (In Chinese).

129. Liu F and Xu RR: Study on the correlation between Chinese medical syndrome types and ID4 gene promoter methylation in human acute myeloid leukemia. Chin J Integr Tradit West Med 32: 471-473, 2012 (In Chinese).

130. Awasthi A, Woolley AG, Lecomte FJ, et al: Variable expression of GLIPR1 correlates with invasive potential in melanoma cells. Front Oncol 3: 225, 2013.

131. Capalbo G, Mueller-Kuller T, Koschmieder S, et al: Endoplasmic reticulum protein GliPR1 regulates $G$ protein signaling and the cell cycle and is overexpressed in AML. Oncol Rep 30 2254-2262, 2013.

132. He Z, Liu H, Agostini M, et al: p73 regulates autophagy and hepatocellular lipid metabolism through a transcriptional activation of the ATG5 gene. Cell Death Differ 20: 1415-1424, 2013.

133. Toska E and Roberts SG: Mechanisms of transcriptional regulation by WT1 (Wilms' tumour 1). Biochem J 461: 15-32, 2014.

134. Dores GM, Devesa SS, Curtis RE, Linet MS and Morton LM: Acute leukemia incidence and patient survival among children and adults in the United States, 2001-2007. Blood 119: 34-43, 2012.

135. Shirley MH, Sayeed S, Barnes I, Finlayson A and Ali R: Incidence of haematological malignancies by ethnic group in England, 2001-7. Br J Haematol 163: 465-477, 2013. 